\title{
Modeling and Simulation of Gear Pumps based on Modelica/MWorks ${ }^{\circledR}$
}

\author{
Chen Liping ${ }^{1}$ Zhao Yan ${ }^{1}$ Zhou Fanli $^{1}$ Zhao Jianjun $^{1}$ Tian Xianzhao ${ }^{2}$ \\ ${ }^{1}$ CAD Center, Huazhong Univ. of Sci. \& Tech., Wuhan, China, 430074 \\ ${ }^{1}$ Suzhou Tongyuan Software \& Control Tech. Co., Suzhou, China, 215123 \\ chenlp@hustcad.comzzhaoyan808@foxmail.com \\ \{fanli.zhou,jjzhao168\}@gmail.com, tianxz@tongyuan.cc
}

\begin{abstract}
In this paper, we present a new method of modeling the hybrid external gear pumps based on Modelica and assess it experimentally. We model the whole working process of an external gear pump. The chamber of the pump is divided into a set of Control Volumes (CVs), whose effective volumes change along with the rotation of the gears. The CVs take in fluid from the inlet port and squeeze fluid out at the outlet port. The whole model of the pump also takes into account flow ripple, pressure distribution, leakages, meshing conditions, etc. Details of each component of the whole pump are provided. From the pressure distribution in space of the gear tooth, we can calculate radial force on shaft, based on which the shaft motion can be simulated.

We carry out a set of predictions in MWorks and report some results on the post processing. These results are consistent with those from the experimental data.
\end{abstract}

Keywords: Hybrid Gear-Pump Modeling; Control Volumes; Pressure Distribution; Radial Force on shaft; Modelica

\section{Nomenclature}

$B$ the gear thickness

$h_{c l}$ the radial clearance of the bearing

$h_{c 2}$ the axial clearance of the bearing

$n$ the tooth number

\section{Introduction}

The gear pumps are among the oldest and most commonly used pumps in the industry. It has become the main choice for fuel system designers due to long life, minimum maintenance, high reliability, capabil- ity to operate with low lubricating fuel, low heat input to fuel, small size, and low weight.

External gear pumps use a simple mechanism (two gears) to generate flow and therefore have a minimum number of parts associated with the design. However, many factors ignored in the design, for example the volumetric efficiency and the pressure peaks, may greatly influence the performance of the pump.

In order to improve the design of the pumps, a good first step is to develop a mathematic model for the simulation. Some work focusing on different aspects of this aim has been done in the past few years.

Manring and Kasaragadda [1] presented an approach to finding a solution for the instantaneous length of action for the two contacting teeth in order to study the flow ripple. In theoretical research, pumps are considered in an ideal case: (1) the fluid is incompressible, (2) fluid leakage is neglected, and (3) the pump parts are inflexible.

Heisler et al [2] used the computational fluid dynamics (CFD) to better analyze the effect of modifying the design of an existing external gear pump. This paper also developed a new approach to simulating the helical gears. Although the computational time and model complexity have been reduced, simulations can be done only under some restrictive assumptions, including restricted flow domain, definite values of the boundary conditions, and fixed position of the gear axes.

Wahab [3] presented a simple analytical and explicit approach to predicting the leakage flow rate under different inlet/outlet pressure differences.

Castilla et al [4,5] studied experimentally the movement of the shaft of a driven gear in a gear pump, in particular the dynamics of the shaft in the journal bearing of a gear pump.

Elia [6] developed a mathematical model that simulates the running in of external gear pumps, taking into account the factors mentioned above. Be- 
sides, the model also estimates the quantity of material taken away.

Following a different way, Vacca et al [7] provided a global description of the pump or motor. They implemented a model, named HYGESim, in the AMESim ${ }^{\circledR}$ platform. The model uses both standard sub-models and $\mathrm{C}++$ sub-models developed by the authors.

Though valuable in some aspects, these studies are unable to develop a global hybrid model, which is the goal of our paper. We construct a hybrid model of the external gear pumps by using a multidomain modeling language, Modelica on MWorks ${ }^{\circledR}$ [8]. The model takes into account the leakages, compressibility of the oil, flow ripple, pressure distribution, etc. It can also calculate forces and torques, which are the base of modeling the shaft motion.

The paper is outlined as follows. Section 2 describes the mechanical system. Section 3 gives an overview of the theory on which the pumps are modeled based and details about each component of the whole pump. Section 4 reports the simulation results and campares them with those in [7]. Section 5 concludes the paper.

\section{Pump Description}

Figure 1 shows a cross-sectional view taken through the gears of a typical gear pump.

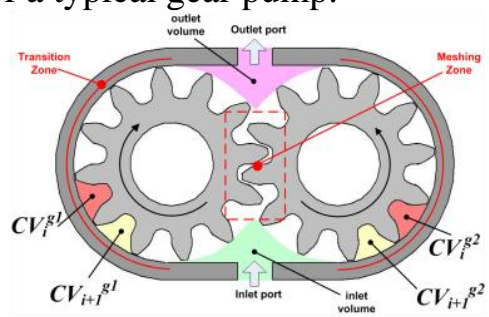

Figure1 Control Volumes Defined in the Fluid Dynamic Model

Note: like most actual gear pumps, this pump has two identical gears to displace fluid. The superscripts g1 and g2 denote the driving and driven gears respectively. The tooth number of gear 1 is the same as that of gear 2. The Control Volumes (CVs) within the tooth gaps are variables dependent on the gear angle. The subscripts $i$ is the index of these Control Volumes. The Outlet and Inlet volumes are two fixed volumes at the outlet and inlet ports respectively.

To produce a flow within a gear pump, fluid is carried by the CVs from the intake side of the pump to the discharge side of the pump through the transition zone. As the gears rotate, these CVs increase their pressure to when reach the high-pressure chamber. As the gear teeth mesh in the meshing zone, fluid is squeezed out of each tooth gap by the mating tooth. When two tooth pairs contact, a trapped volume is generated. This may cause positive pressure peaks and the onset of cavitation. (To avoid this, the trapped volume must be connected to the high or low pressure chambers, which is the role of the relief grooves in the lateral bushes.) On the intake side, the gear teeth are coming out of the mesh. The volumes of CVs increase so that fluid is inhaled into the tooth gaps. This process repeats itself for each revolution of the pump and therefore displaces fluid at a rate proportional to the pump speed.

\section{Model Description}

\subsection{Overview}

In this section, we first give an overview of the pump model. Some components come from a free library HyLibLight_[9] based on Modelica.

The aim of this paper is to construct a hybrid model of the external gear pumps. It considers the leakages, compressibility of the oil, flow ripple, pressure distribution, etc. Specifically, the pump is modeled under the following assumptions:

1) The gears and the housing case are rigid; only the oil is compressible.

2) The position of the shaft is known before the simulation and is fixed during the simulation.

3) The pressure at every single isolated region and fixed volume is well-proportioned.

4) The temperatures in all CVs are the same and constant.

5) The tooth numbers of the two gears are the same.

In Figure1, a pump is divided into $2 n+2$ control volumes, where $n$ is the tooth number of a gear. Figure 2 shows the flows between those control volumes. This method is similar to but not the same as that in [6]. There is no variation in the number of the control volumes which causes variation in the number of differential equations. 


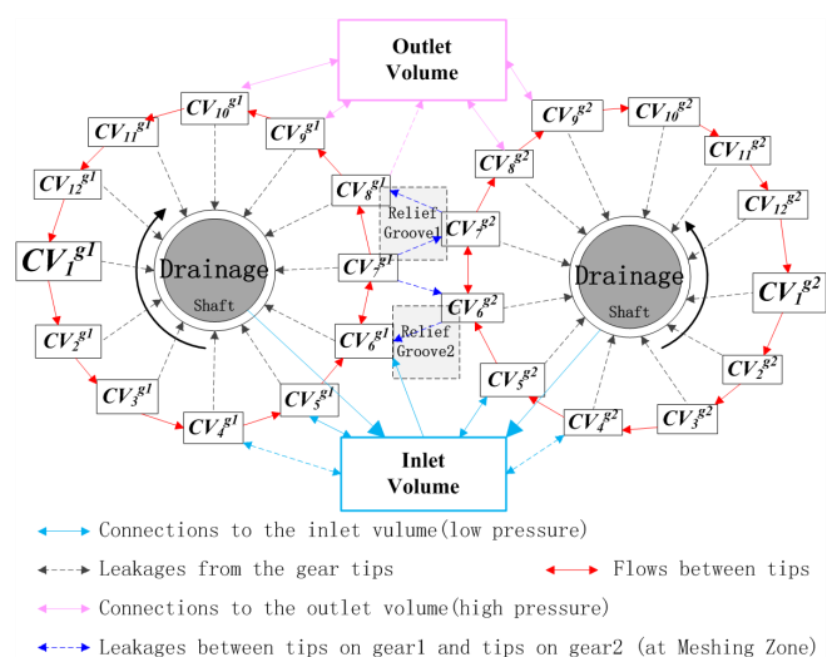

Figure 2 The Flows Between the Control Volumes

Note: arrows do not mean the flow directions; the direction of the flow depends on the pressure differential and the rotation direction. As the gears rotating, the channels of some flows are shutting down and some of others (not shown in this figure) are turning on.

Dark blue lines in the meshing zone represent the leakages from a trapped volume on one gear to a $\mathrm{CV}$ on the other gear.

The flow between $C V_{i}^{g 1}$ and $C V_{i+1}^{g 2}$ is the leakage through the clearance between two meshing surfaces;

The flow between $C V_{i}^{g 1}$ and $C V_{i}^{g 2}$ is the leakage through the clearance between two un-meshing surfaces.

Relief Grooves are chambers to which the CVs connect in turn. Flows from the CVs to the Relief Grooves in the meshing zone are shown in Figure 3.

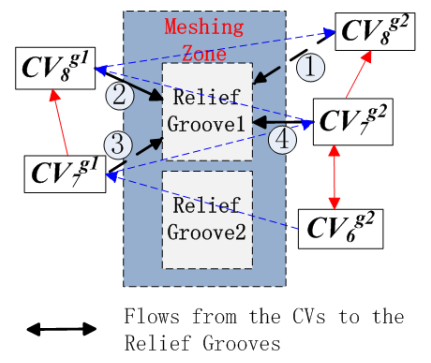

Figure 3 Flows from the CVs to the Relief Grooves (RG)

RG1 on the outlet side is used to decrease the peak of the pressure trapped in the CVs; RG2 on the inlet side is used to restrain the cavitation.

As shown in Figure 3, the dashed line 1 means the flow between $C V_{8}{ }^{g 2}$ and RG1 is forming. Solid line 2 and 4 mean that there is a fluid flow between $C V_{8}{ }^{g 1}$ and $C V_{7}{ }^{g 2}$ through RG1. Dashed line 3 means that the channel between $C V_{7}^{g l}$ and RG1 is being turned off.

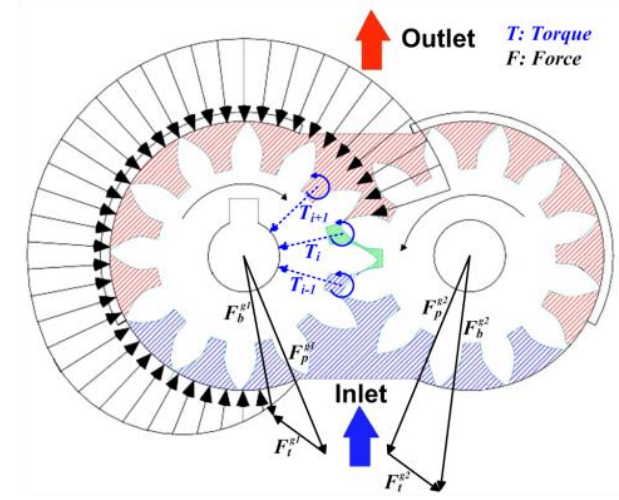

Figure 4 Calculated Forces \& Torques Acting on Gear 1

Figure 4 shows the calculated Forces and Torques acting on the driving gear, where $F_{p}{ }^{g l}$ is the resultant force of the pressure distributed along the circle, $F_{c}{ }^{g l}$ is the contact force, $F_{b}{ }^{g l}$ is the force load on the bearing of gear 1 and $T_{i}$ is the torque supplied by the pressure in $\mathrm{CVi}$.

Note: most of CVs, except those in the meshing zone, do not have any effect on the gear rotation, because the pressures in these tooth gaps counteract and no torque is acting (for example, $\mathrm{T}_{\mathrm{i}+1}=0$ ). Details will be given in the next few sections.

The coordination system is shown in Figure 5.

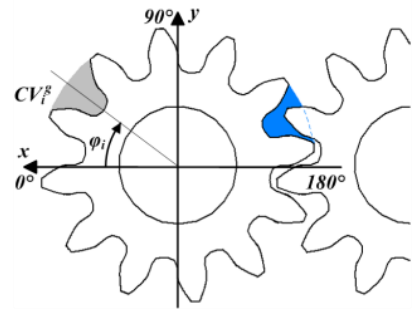

Figure 5 Coordination System

\subsection{Control Volumes}

\subsubsection{Volume}

A Control Volume is the space between two adjacent teeth. The maximum volume (see Figure 5, the gray region) of this gap is related to the gear dimensions (e.g. tooth number, modulus, thickness, etc). As the gears rotating, gear pairs mesh together. A tooth on the other gear squeezes into the gap, so the effective volume of the gap decreases (see Figure 5, the blue region). The effective volume of $\mathrm{CVi}$ is a function of the angle $\varphi_{i}$ :

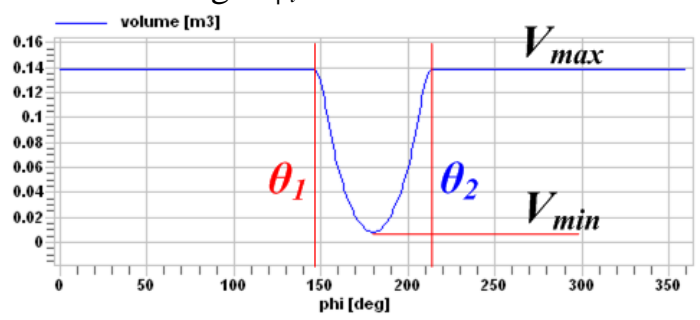

Figure 6 Effective Volume as a Function of Angle $\varphi$ 
This relationship can be expressed in an approximate equation:

$$
V=\left\{\begin{array}{l}
V_{\max }, \quad \varphi<\pi-\theta_{1} \text { or } \varphi>\pi+\theta_{2} \\
x_{3}\left[x_{2} x_{1}\left(V_{\max }-V_{\min }\right) / 2 \times\right. \\
\left.\left.\cos ((\varphi-\pi) f)+\left(V_{\max }+V_{\min }\right) / 2\right)\right]
\end{array},\right. \text { otherwise }
$$

where

$$
\begin{aligned}
x_{3} & =c_{31} \cos ((\varphi-\pi) f)+c_{32} \\
x_{2} & =c_{21} \cos ((\varphi-\pi) f)+c_{22} \\
x_{1} & =c_{11} \cos ((\varphi-\pi) \times 2 f)+c_{12} \\
f & =2 \pi /\left(\theta_{1}+\theta_{2}\right) \\
c_{11} & =0.25 \quad c_{12}=0.75 \quad c_{21}=0.25 \\
c_{22} & =-0.75 \quad c_{31}=-0.4 \quad c_{32}=0.6 \\
\text { phase } & =\left\{\begin{array}{cc}
2 \pi / n \times(\text { Index }-1) & \text {,if gear } 1 \\
2 \pi / n \times(\text { Index }-0.5) & \text {,if not }
\end{array}\right.
\end{aligned}
$$

$\theta_{1}$ and $\theta_{2}$ are the critical angles (see Figure 7).

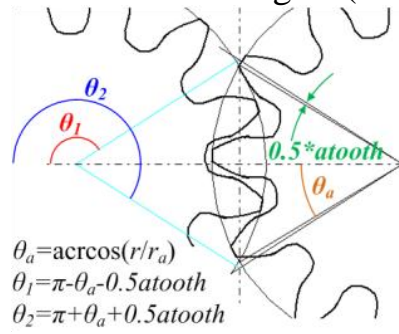

Figure 7 Critical Angles

\subsubsection{Pressure distribution}

The pressure in each $\mathrm{CVi}$ is given by the equation:

$$
\dot{p}=\frac{\beta}{V} \times\left(q+q_{\text {cham }}\right)
$$

where

\section{$B$ oil bulk modulus}

$q_{\text {cham }}$ the rate of the net flow that flows into the chamber

$$
p \text { pressure of the volume }
$$

The pressure distribution is shown in Figure 4.

\subsubsection{Radial Force Produced by Pressure}

As shown in Figure 4, the load on a bear is the sum of the loads produced by the distributing pressure and the contact force. Ideally, the loads due to the pressure of most CVs have only one radial component ( $F_{r}$ in Figure 4), since we have assumed (in section 错误!未找到引用源。) that the pressure in any isolated region is well-proportioned.

When a CV turns into the meshing zone, the chamber will be divided into 2 or 3 parts by the coupling tooth on the other gear (see Figure 8). The load due to the pressure in red zone 1 is equal to the sum of $F_{t}$ and $F_{r}$. And

$$
\begin{aligned}
& F_{t 1}=B \times P_{i}^{g 2} \times\left(r_{m}-r_{a}\right) \\
& F_{r 1}=B \times P_{i}^{g 2} \times\left(r_{m} * \gamma_{1}\right)
\end{aligned}
$$

Similarly, for zone 2 and zone 3 we have:

$$
\begin{aligned}
& F_{t 2}=B \times P_{i}^{g 1} \times\left(r_{n}-r_{m}\right) \\
& F_{r 2}=B \times P_{i}^{g 1} \times\left(r_{m} * \gamma_{2}\right) \\
& F_{t 3}=B \times P_{i-1}{ }^{g 2} \times\left(r_{a}-r_{n}\right) \\
& F_{r 3}=B \times P_{i-1}{ }^{g 2} \times\left(r_{n} * \gamma_{1}\right)
\end{aligned}
$$

Then:

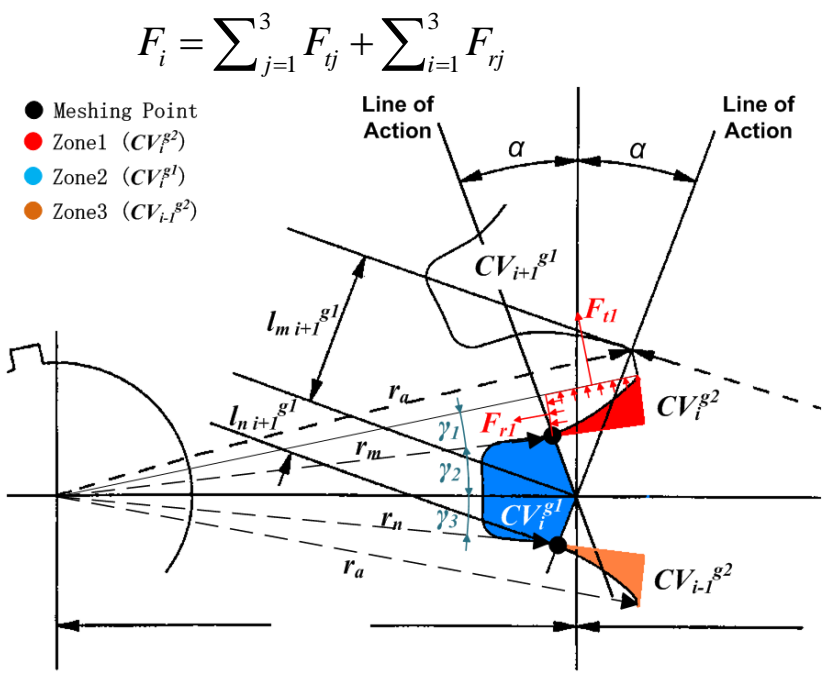

Figure 8 The chamber of $C V_{i}^{g 1}$ divided into 3 parts

\subsubsection{Torque}

Because only the component Ft has an effect on the gear rotation, the torque produced by $C V_{i}^{g l}$ can be calculated as follows:

$$
T=\left(F_{t 1}\left(r_{a}+r_{m}\right)+F_{t 2}\left(r_{m}+r_{n}\right)+F_{t 1}\left(r_{n}+r_{a}\right)\right) / 2
$$

\section{Interfaces and Icon}

The interfaces and icon are shown in Figure 9.

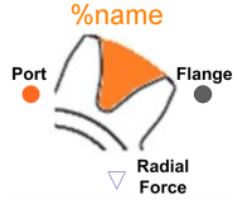

Figure 9 Icon of a Control Volume

\subsection{Internal Leakages}

Both gears rotate within the housing. The interspace is made because of lubrication between two relative moving objects. The gap between two gear teeth and casing is assumed to maintain a known value. Leakage occurs through these internal clearances due to both the pressure differences and the relative motion. 
There are four types of sources of internal leakages due to the pressure differences:

$Q_{p}$ : Flows from one $\mathrm{CV}$ to an adjacent one through the tips of the gear teeth and the case.

$Q_{z}$ : Flows from the outlet volume to the inlet volume through the meshing zone.

$Q_{m}$ : Flows through the gap between two mating surfaces.

$Q_{d}$ : Flows from the CVs to the drainage circle through the interspace between the gear and the case.

There is another kind of leakage that is caused by the relative motion and oil viscosity (see Figure 10, $Q_{w}$ ). It results in a little increase in the flow rate because the rotation brings oil to the outlet volume.

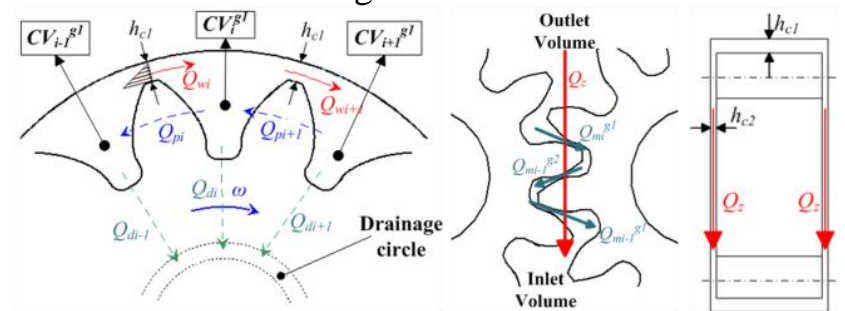

Figure 10 Internal Leakages

In general, for the first type of leakage it is assumed that the pressure around the case rises linearly across each tooth tip. Hence, the volumetric flow from $C V_{i}^{g l}$ to $C V_{i-1}{ }^{g l}$ due to the pressure drop is given by ([2]):

$$
Q_{p i}=d p \times G=d p \times h^{3} b /(6 \rho v \sqsubset r)
$$

where $d p$ is the pressure differential. 11.

The diagram of leakage QP is shown in Figure

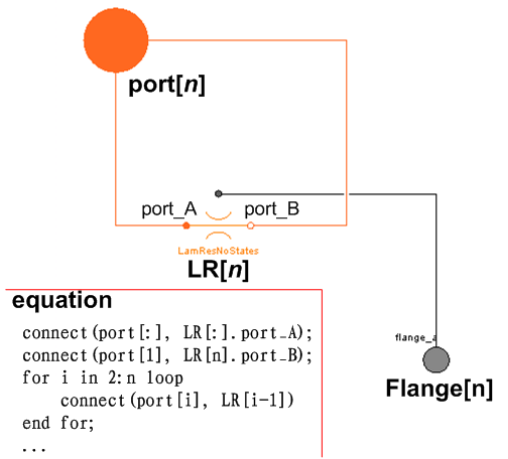

Figure 11 Diagram of Leakage $Q_{p}$

where $n$ is the tooth number, LR is a laminar resistor model [9].

Other leakages due to pressure are similar to $Q_{p i}$, see [2][4].

The volumetric flow produced by the relative motion has a uniform distribution from zero to $v$, where $v$ is the relative velocity of the tip with respect to the case. The volumetric flow is defined as [2]:

$$
Q_{w i}=h_{c 1} \times B \times v / 2
$$

The interfaces and icons of five types of leakages are shown in Figure 12.

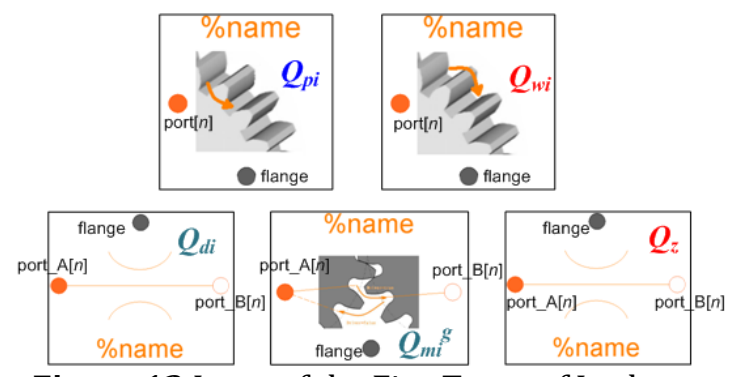

Figure 12 Icons of the Five Types of Leakages

\subsection{Mechanical Parts}

Mechanical parts contain the mounting, the bears and the mesh models. Mounting is used to fix the bears that support the gear pair. This paper does not cover the position of the shaft. So we choose a component FixedTranslation from Modelica.Mutibody to fix the two gears. Model IdealGear is used to transmit torque, which does not consider elasticity, damping or backlash. If we want to consider these effects, the gears have to be connected to other elements in an appropriate way.

The diagram of mechanical parts is shown in Figure 13.

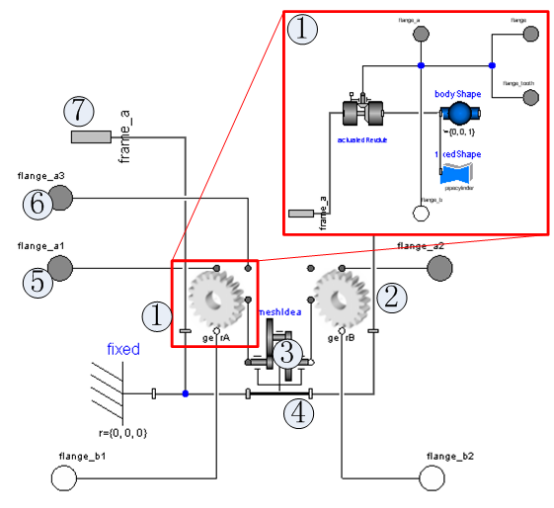

Figure 13 Diagram of the Mechanical Parts

1-Driving gear 2-Driven gear 3-IdealMesh 4-

FixedTranslation 5-flange that transmits a driving torque

6-flange that transmits the resistance moment 7frame, forces on shaft 1

Interfaces and icon of the mechanical parts are shown as follows:

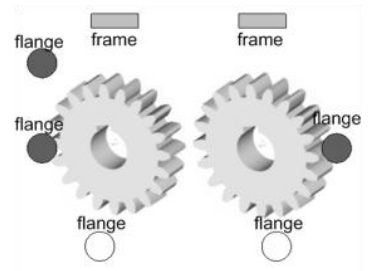

Figure 14 Interfaces and Icon of the Mechanical Parts 


\subsection{Radial Forces}

Radial Forces produced by the CVs has been calculated (see section 3.2.3). In the model of CV, radial force is decomposed into $\mathrm{x}$ - and $\mathrm{y}$-components, which are then summed up in the model RadialForce. Figure 15 shows the diagram of the RadialForce as well as its interfaces and icon.

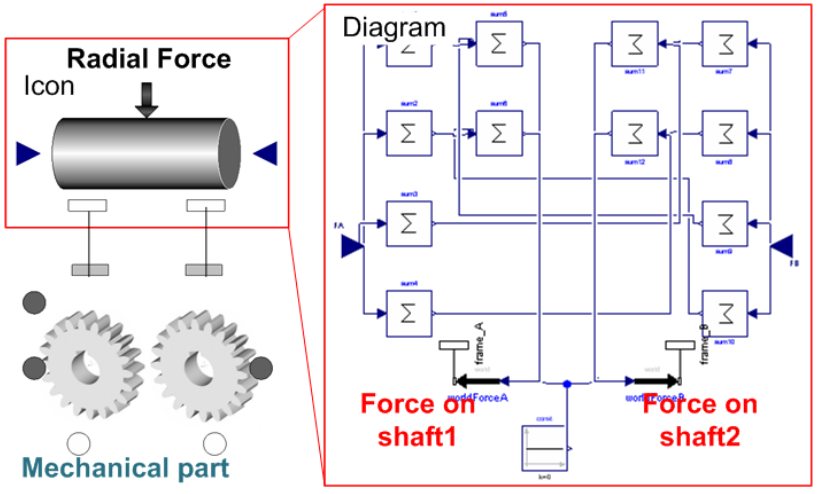

Figure 15 Interfaces, Icon and Diagram of Model RadialForce

\subsection{Channels from CVs to Inlet/Outlet Volumes}

Along with the rotation of the gears, when a CV comes to the position of $\mathrm{CV}_{i+2}{ }^{g l}$, the channel between it and the outlet volume opens; when it comes to the position of $C V_{i}^{g l}$, this channel becomes to close. Critical angles, shown in Figure 16, are calculated in respective models.

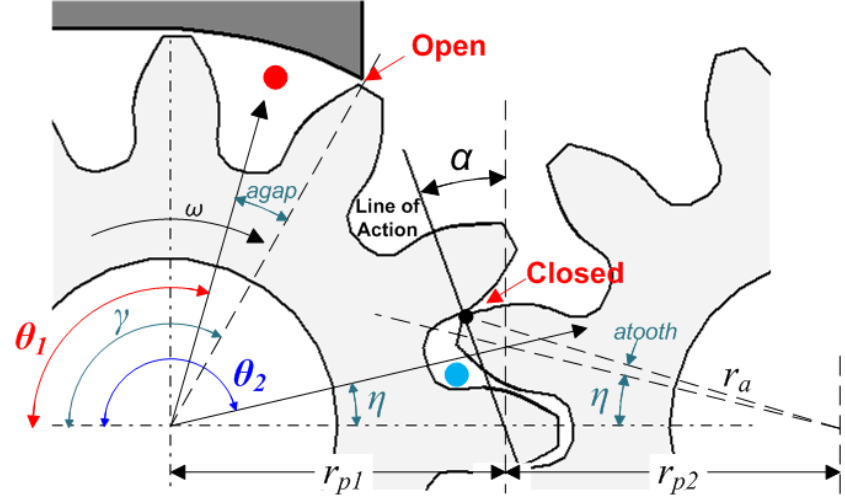

Meshing Point Zone1 $\left(\boldsymbol{C V}_{\boldsymbol{i + 2}}{ }^{g I}\right) \bigcirc$ Zone2 $\left(\boldsymbol{C} \boldsymbol{V}_{i}^{g I}\right)$

Figure 16 Critical Angles of Channel Model Connecting $\mathrm{CV}$ and Outlet Volume

Equations of the critical angles are given as follows:

$$
\begin{aligned}
& \theta_{1}=\gamma+a_{g a p} \\
& \theta_{2}=\pi-\eta
\end{aligned}
$$

where $\gamma$ is a parameter about the pump dimension. $\eta$ can be calculated from Figure 16.

The hydraulic diameter of the channel connecting a $\mathrm{CV}$ to the outlet volume is given by:

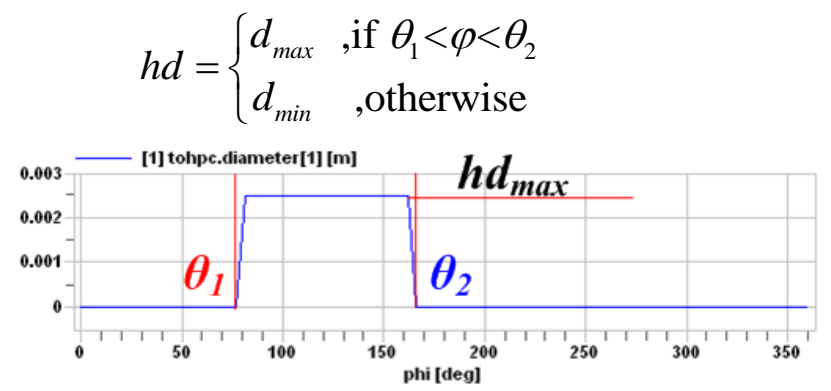

Figure 17 Hydraulic-Diameter as a Function of Angle $\varphi$

Interfaces and icons of a channel are shown in Figure 18.

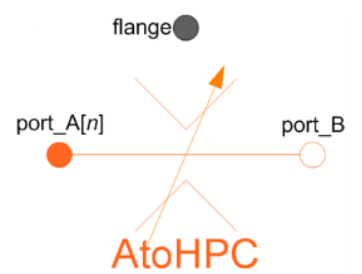

Figure 18 Interfaces and Icon of a Model connected to Outlet Volume (High Pressure Chamber)

The channel between a CV and the inlet volume is similar.

\subsection{Viscosity Torque}

In the ideal case, Viscosity models can be treated as dampers. There are two types of viscosity torques acting on a gear, the radial torque and the axial torque (see Figure 19). The equations of these torques are given by:

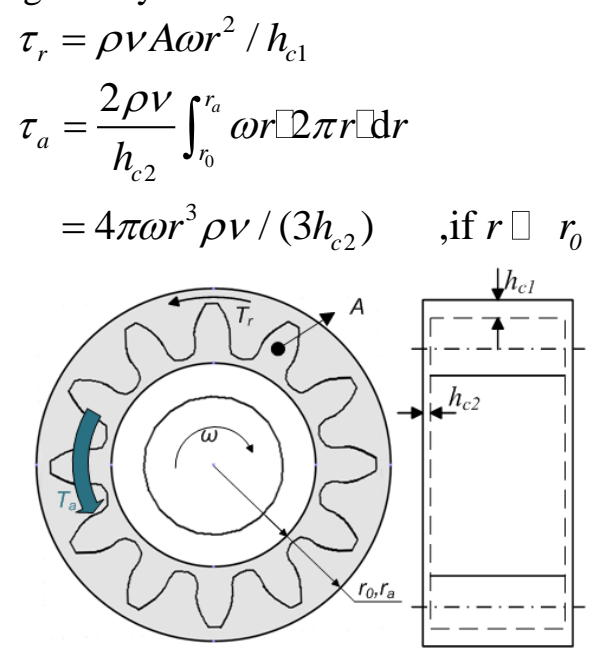

Figure 19 Radial Torques and Axial Torques

Interfaces and icons of the torque models are shown in Figure 20. 


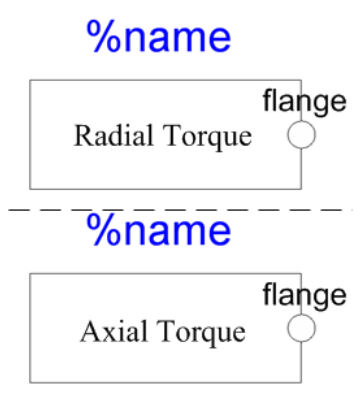

Figure 20 Interfaces and Icons of Torque Models

Other resistance moments, for example those caused by the bears, can be added as a component.

\subsection{System Model}

Combining all the components above, we obtain a whole pump as follows:

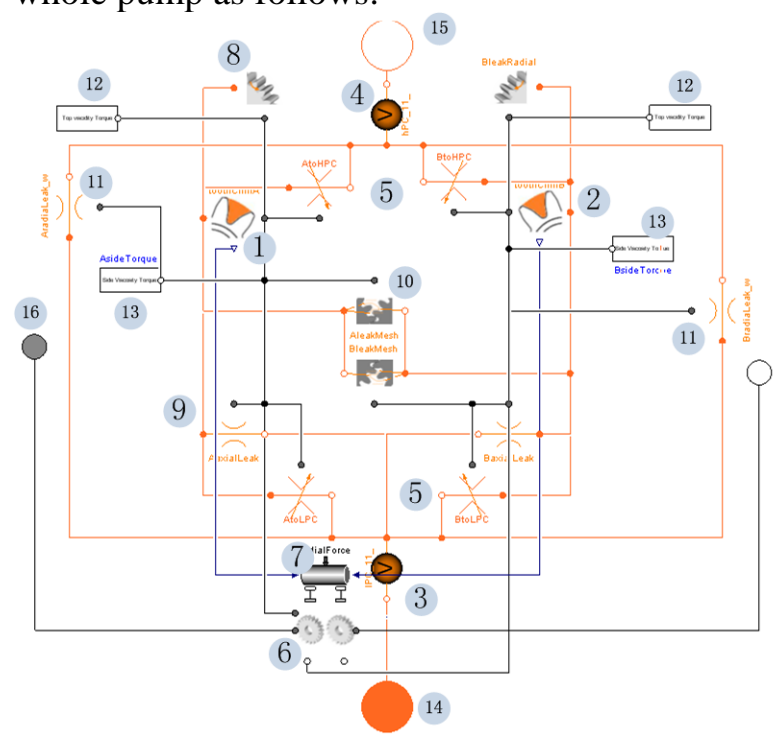

1-CVs on gear1 2-CVs on gear2 3-inlet vol 4-outlet vol 5-Channels(to in/outletChm) 6-Mechanical part 7-adialForce(optional) 8 11-Leakages 12 13-ViscocityTorques

14-inlet port 15-outlet port 16-shaft of gear1

Figure 21 Diagram of a Whole Pump

The interfaces and icon are shown in Figure 22.

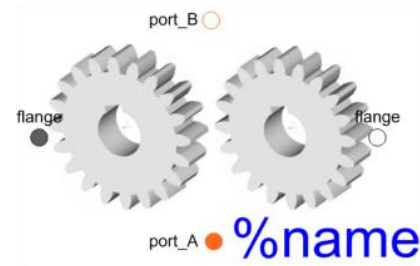

Figure 22 Interfaces and Icon of a Pump Model

\section{Simulation Results}

We implement the simulation based on the model shown in Figure 23 to enable the comparison between our results and the experimental results in [7].

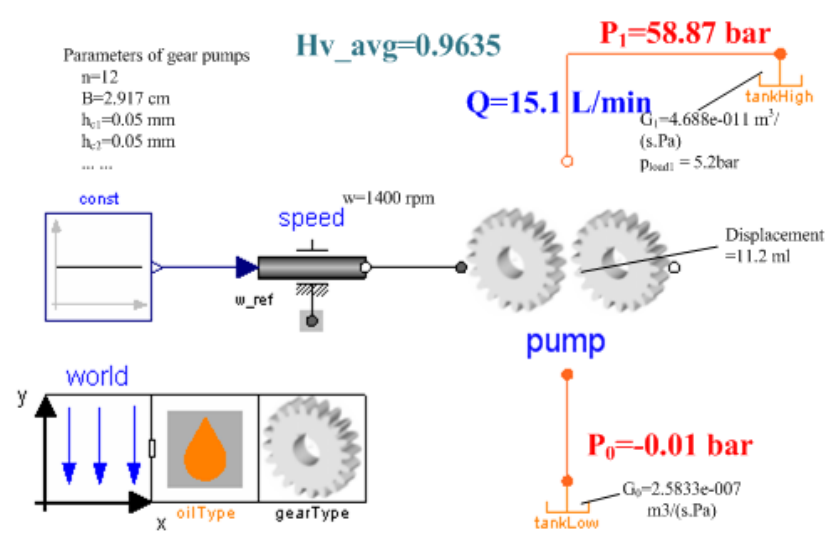

Figure 23 Simulation system diagram

The simulation curves of the volumetric flow ripple as well as the average flow rate (in L/min) is shown in Figure 24.

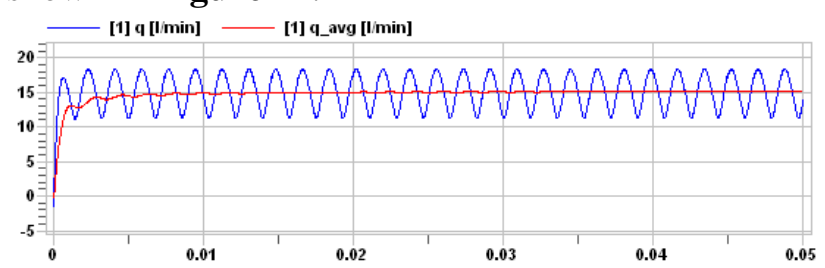

Figure 24 Volumetric flow ripple and average flow rate

The pressure distribution in a tooth space within a complete rotation cycle is shown in Figure 25.

Note: the pressure distribution in Figure $\mathbf{2 5}$ is very similar to the pressure distribution along the circle of a gear. But actually they are not the same.

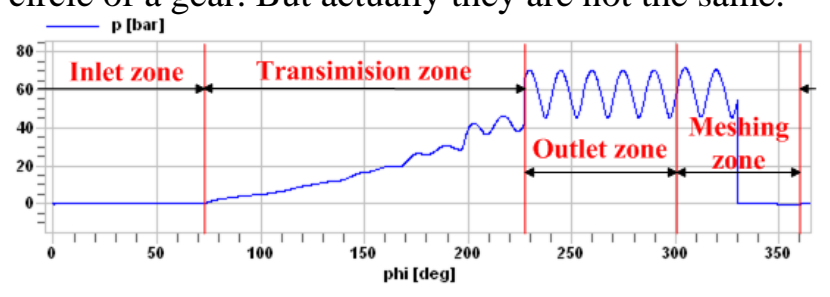

Figure 25 Pressure Distribution in a Tooth Space for a Complete Rotation Cycle

Order:

$$
\begin{aligned}
& p_{x}=-(P+1 \mathrm{e} 6) \times \cos (\varphi) \\
& p_{y}=(P+1 \mathrm{e} 6) \times \sin (\varphi)
\end{aligned}
$$

The pressure distribution above is shown in Figure 26.
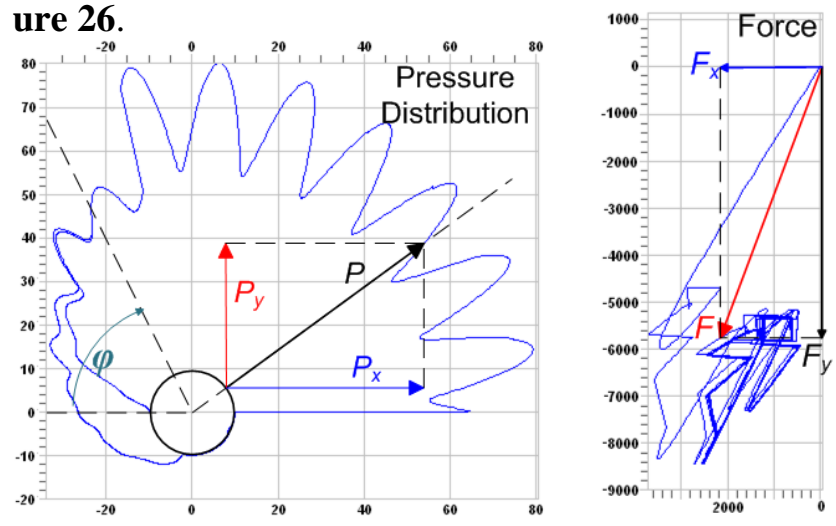
Figure 26 (Left) Pressure distribution in a Tooth Space for a Complete Rotation Cycle bars.

The radius of the unit circle at the bottom is 10

Figure 27 (Right) Radial Force on Shaft 1 for a Complete Rotation Cycle and its Components in the Coordination in Figure 5.

By changing the parameters (e.g. $G_{0}, G_{1}$ shown in Figure 23), we obtain a set of predictions. Both the simulation results and the experimental results from [7] are listed in Figure 28Figure 29. It is clear that the simulation and the experimental results are very close.

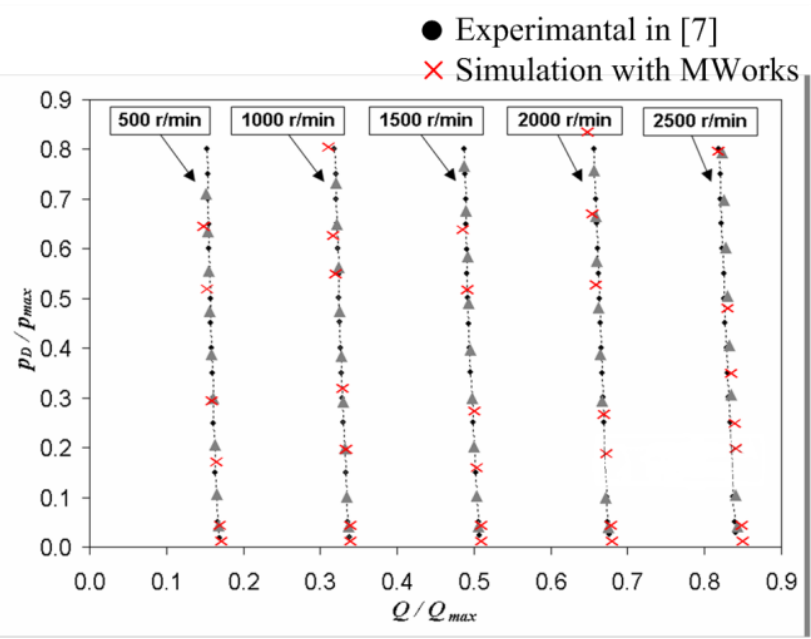

Figure 28 Dimensionless Characteristics of the Pump in Steady States

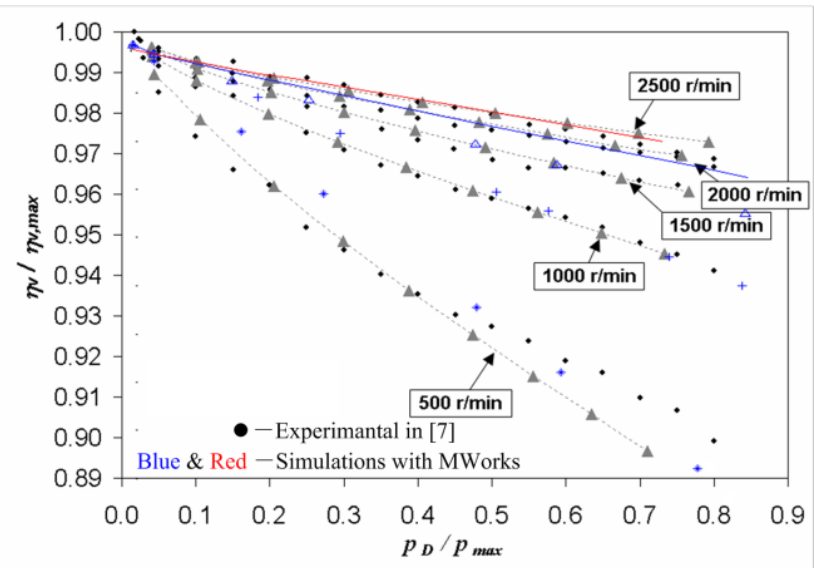

Figure 29 Dimensionless Values of the Volumetric Efficiency of the pump in Steady Conditions

\section{Conclusions and Future Work}

We present a new method of modeling the hybrid external gear pumps based on Modelica and assess it via simulation. The model takes into account the flow ripple, pressure distribution, leakages, meshing conditions, etc. Details of the components that make up the whole pump are introduced. Comparisons be- tween the result and the experimental data are given in the end. Good agreement between them is founded.

From the pressure distribution in the space of a gear tooth, radial force on shaft can be easily calculated, on which the motion of the shaft can be simulated based. This is left for future work.

Another weakness of the study is that there is little comparison with real applications and measurement data. It is a drawback as some minor malfunctions and parameters probably could not be identified. Such comparison is also left for future work.

\section{Acknowledgments}

The paper is supported by National Nature Science Foundation of China (No.60874064, No.60704019, No.60736019), Key Project of National High Technology Research and Development Program (2009AA044501).

\section{References}

[1] Noah D.Manring, Suresh B.Kasaragadda. The Theoretical Flow Ripple of an External Gear Pump. Journal of Dynamic Systems, Measurement, and Control. Transaction of the ASME 2003, September: 396-404

[2] Aaron S.Heisler, John J.Moskwa, Frank J.Fronzak. SIMULATED HELICAL GEAR PUMP ANALYSIS USING A NEW CFD APPROACH. Fluids Engineering Division Summer Meeting 2009-78472

[3] Abdul Wahab. ANALYTICAL PREDICTION TECHNIQUE FOR INTERNAL LEAKAGE IN AN EXTERNAL GEAR PUMP. Proceedings of ASME Turbo Expo,GT 2009-59287

[4] R.Castilla, M.Gutes, P.J.Gamez-Monter, E.Codina. Experimental Study of the Shaft Motion in the Journal Bearing of a Gear Pump. Journal of Engineering for Gas Turbines and Power, 2009-052502

[5] R.Castilla, M.Gutes, P.J.Gamez-Monter, E.Codina. Numerical Analysis of the Shaft Motion in the Journal Bearing of a Gear Pump. Journal of Engineering for Gas Turbines and Power, 2010-012504

[6] G.Dalpiaz, G.D’Elia, E.Mucchi, A.Fernandez der Rincon. MODELING RUN IN PROCESS IN EXTERNAL GEAR PUMPS. Proceedings of ESDA2006-95466

[7] Andrea Vacca, Germano Franzoni and Paolo Casoli. ON THE ANALYSIS OF EXPERIMENTAL DATA FOR EXTERNAL GEAR MACHINES AND THEIR COMPARISON 
WITH SIMULATION RESULTS. International Mechanical Engineering Congress and Exposition, IMECE2007-422664

[8] Fan-Li Zhou, Li-Ping Chen, Yi-Zhong Wu, Jian-Wan Ding, Jian-Jun Zhao, Yun-Qing Zhang. MWorks: a Modern IDE for Modeling and Simulation of Multidomain Physical Systems Based on Modelica. Modelica 2006, September 4th - 5th: 725-732

[9] P.Beater. Modeling and Digital Simulation of Hydraulic Systems in Design and Engineering Education using Modelica and HyLib. Modelica Workshop 2000 Proceedings:33-40 